HEPHY-PUB 1012/18

December 2018

\title{
THE SPINLESS RELATIVISTIC HELLMANN PROBLEM
}

\author{
Wolfgang LUCHA* \\ Institute for High Energy Physics, \\ Austrian Academy of Sciences, \\ Nikolsdorfergasse 18, A-1050 Vienna, Austria \\ Franz F. SCHÖBERL ${ }^{\dagger}$ \\ Faculty of Physics, University of Vienna, \\ Boltzmanngasse 5, A-1090 Vienna, Austria
}

\begin{abstract}
We compile some easily deducible information on the discrete eigenvalue spectra of spinless Salpeter equations encompassing, besides a relativistic kinetic term, interactions which are expressible as superpositions of an attractive Coulomb potential and an either attractive or repulsive Yukawa potential and, hence, generalizations of the Hellmann potential employed in several areas of science. These insights should provide useful guidelines to all attempts of finding appropriate descriptions of bound states by (semi-) relativistic equations of motion.
\end{abstract}

PACS numbers: 03.65.Pm, 03.65.Ge, 12.39.Pn, 11.10.St

Keywords: relativistic bound states, Bethe-Salpeter formalism, spinless Salpeter equation, Rayleigh-Ritz variational technique, generalized-Hellmann potentials, critical parameters.

* E-mail address: Wolfgang.Lucha@oeaw.ac.at

$\dagger$ E-mail address: franz.schoeberl@univie.ac.at 


\section{Spinless-Salpeter-Based Approach to Bound States}

Within quantum field theory, the homogeneous Bethe-Salpeter equation [1-3] constitutes a Poincaré-covariant approach to bound states. Driven by the desire to describe bound states to the utmost reasonable extent by analytic tools, a variety of directions has been proposed for the diminution of the complexity inherent to the Bethe-Salpeter formalism. Performing a three-dimensional reduction, by assuming for the involved bound-state constituents both propagation like free particles and instantaneity of their mutual interactions, and dropping any negative-energy contribution and all bound-state constituents' spin degrees of freedom takes us to the spinless Salpeter equation: a semirelativistic bound-state equation that may be formulated as the eigenvalue equation of an appropriate Hamiltonian $H$ composed of its relativistic kinetic energy $T$ and some interaction potential $V$. For bound states of only two constituents of relative momentum $\boldsymbol{p}$, relative coordinate $\boldsymbol{x}$, and masses $m$, for simplicity of notation here chosen to be equal, this operator $H$ is (in natural units $\hbar=c=1$ ) of the form

$$
H \equiv T(\boldsymbol{p})+V(\boldsymbol{x}), \quad T(\boldsymbol{p}) \equiv 2 \sqrt{\boldsymbol{p}^{2}+m^{2}} .
$$

This class of Hamiltonians may be viewed as immediate generalization of its nonrelativistic Schrödinger counterpart towards incorporation of relativistic kinematics, albeit deduced at the price of having to cope with the nonlocality induced by the "relativized" kinetic energy.

The nonlocality of any semirelativistic Hamiltonian $H$ such as the one of Eq. (1) poses a grave obstacle to the preferred analytic solvability of the corresponding equation of motion. Consequently, when attempting to pin down the solutions to such bound-state equation by whatever approach seems best to one, reliable guidelines, in form of rigorous constraints on any bound-state spectrum to be expected, should be highly welcome. Needless to say, upon having a set of solutions at one's disposal, its significance can be easily examined by various really powerful tools, for instance, the generalization of the virial theorem of nonrelativistic quantum theory to the incorporation of relativistic kinematics [4,5]. Utilizing techniques of this kind has proven extremely efficient in the separation of the wheat from the chaff [6-10].

In the present analysis, we adopt various considerations of the above type (to the extent applicable) for a characterization of what can be dubbed the relativistic Hellmann problem, obtained by specifying the interaction term $V(\boldsymbol{x})$ in our Hamiltonian (1) to be a spherically symmetric potential $V(r)(r \equiv|\boldsymbol{x}|)$ of a form that generalizes the shape originally proposed by Hellmann $[11,12]$ and that has found widespread application in different areas of physics and chemistry. By definition, that generalization of Hellmann's potential is a superposition

$$
\begin{aligned}
V(\boldsymbol{x})=V_{\mathrm{H}}(r) & \equiv V_{\mathrm{C}}(r)+V_{\mathrm{Y}}(r) \\
& =-\frac{\kappa}{r}-v \frac{\exp (-b r)}{r}, \quad \kappa \geq 0, \quad v \gtreqless 0, \quad b>0,
\end{aligned}
$$

of a, by convention, attractive Coulomb term $V_{\mathrm{C}}(r)$ with related coupling parameter $\kappa \geq 0$,

$$
V_{\mathrm{C}}(r)=-\frac{\kappa}{r}, \quad \kappa \geq 0
$$

and a Yukawa term $V_{\mathrm{Y}}(r)$ with related coupling strength $v \gtreqless 0$ and range parameter $b>0$,

$$
V_{\mathrm{Y}}(r)=-v \frac{\exp (-b r)}{r}, \quad v \gtreqless 0, \quad b>0 .
$$

In this spirit, after listing all possible shapes of $V_{\mathrm{H}}(r)$ (Sec. 2), we explore the circumstances under which $H$ is bounded from below (Sec. 3), evaluate our chances of limiting the number of bound states (Sec. 4), constrain their energies (Sec. 5), and reassess our findings (Sec. 6). 


\section{Classification: Possible Hellmann-Potential Shapes}

Let us start by examining the gross behaviour of our potential $V_{\mathrm{H}}(r)$ for $r \rightarrow \infty$ and $r \rightarrow 0$.

- In the limit $r \rightarrow \infty$, any Yukawa potential $V_{\mathrm{Y}}(r)$ vanishes, due to its rapidly decaying exponential factor, faster than the Coulomb potential $V_{\mathrm{C}}(r)$. Accordingly, the large- $r$ behaviour of the Hellmann potential $V_{\mathrm{H}}(r)$ inevitably approaches that of its Coulomb component $V_{\mathrm{C}}(r)$, independently of the values of the Yukawa parameters $v$ and $b>0$ :

$$
V_{\mathrm{H}}(r) \underset{r \rightarrow \infty}{\longrightarrow}-\frac{\kappa}{r} \equiv V_{\mathrm{C}}(r)
$$

- The $V_{\mathrm{H}}(r)$ behaviour near the origin is determined by the size of $v$ relative to $-\kappa \leq 0$ :

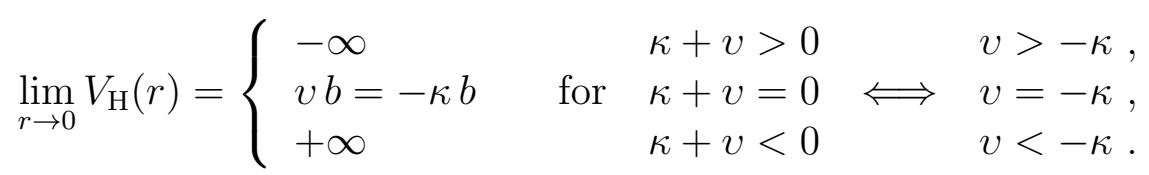

There exist some further peculiar features of the Hellmann potential (2) worth mentioning:

- The case of sufficiently negative Yukawa coupling, more exactly, the interval $v<-\kappa$, defines the only instance where $V_{\mathrm{H}}(r)$ develops a repulsive core, enabled by its zero at

$$
r_{0}=-\frac{1}{b} \ln \left(-\frac{\kappa}{v}\right)=\frac{1}{b} \ln \left(-\frac{v}{\kappa}\right)
$$

and, accordingly, a global minimum $V_{\mathrm{H}}(\underline{r})$, situated at the straightforward solution of

$$
v(1+b \underline{r}) \exp (-b \underline{r})=-\kappa \text {. }
$$

- For sufficiently positive Yukawa coupling, that is to say, for $v>\kappa$, we find a crossover of the Coulomb and Yukawa contributions to the Hellmann potential for finite $r$, viz.,

$$
r_{\times}=-\frac{1}{b} \ln \frac{\kappa}{v}=\frac{1}{b} \ln \frac{v}{\kappa} .
$$

In more detail, we may classify the behaviour of the Hellmann potential into six intervals of the Yukawa coupling $v$ (leaving aside the value $v=0$ reducing $V_{\mathrm{H}}(r)$ to the Coulomb case):

$$
\begin{aligned}
& v>\kappa \quad \Longrightarrow \quad \kappa+v>0, \\
& v=\kappa \quad \Longrightarrow \quad \kappa+v>0 \text {, } \\
& 0<v<\kappa \quad \Longrightarrow \quad \kappa+v>0, \\
& -\kappa<v<0 \quad \Longrightarrow \quad \kappa+v>0 \text {, } \\
& v=-\kappa \quad \Longrightarrow \quad \kappa+v=0 \text {, } \\
& v<-\kappa \quad \Longrightarrow \quad \kappa+v<0 \text {. }
\end{aligned}
$$

Figure 1 shows an illustrative example for each distinct potential shape, with the plots 1(a) through 1(f) corresponding bijectively to the $v$ regions (3a) through (3f). For a vanishing Yukawa coupling, the Hellmann potential reduces, trivially, to the Coulomb potential. The spinless relativistic problem posed by the latter has been analyzed very extensively [13-17]. 


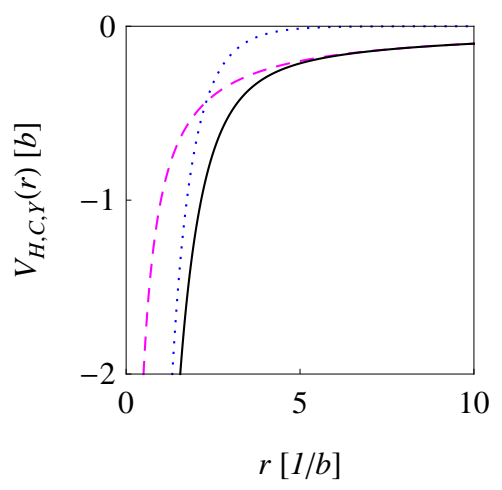

(a)

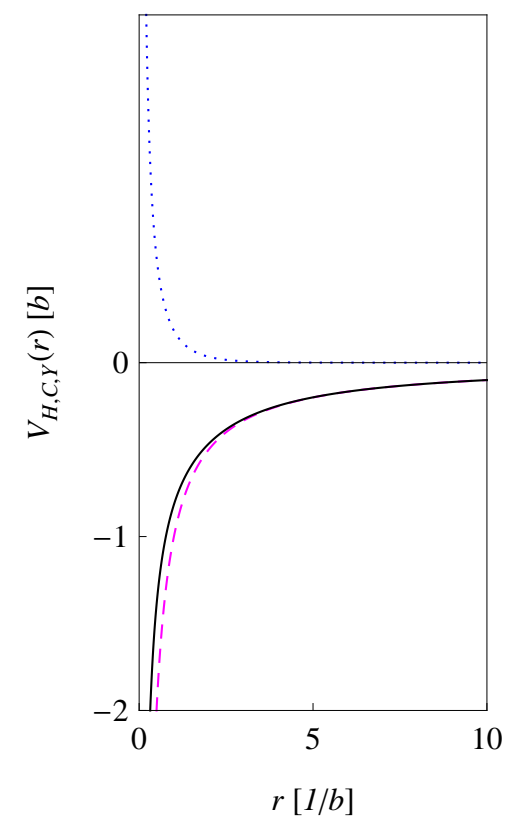

(d)

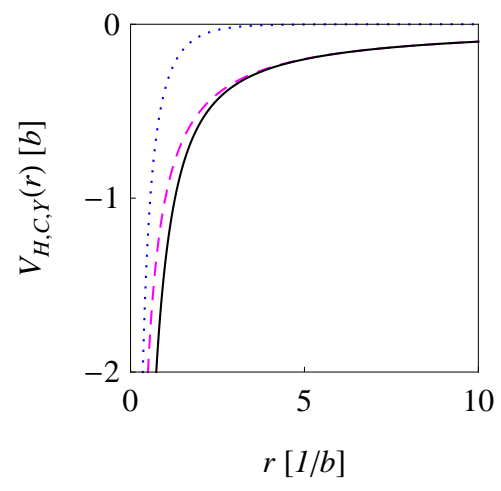

(b)

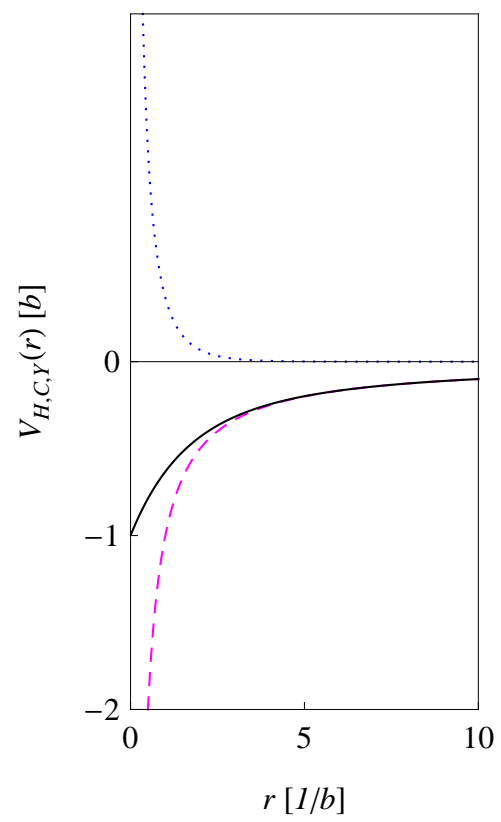

(e)

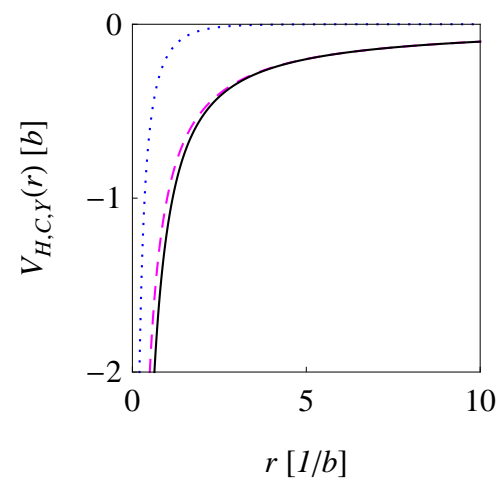

(c)

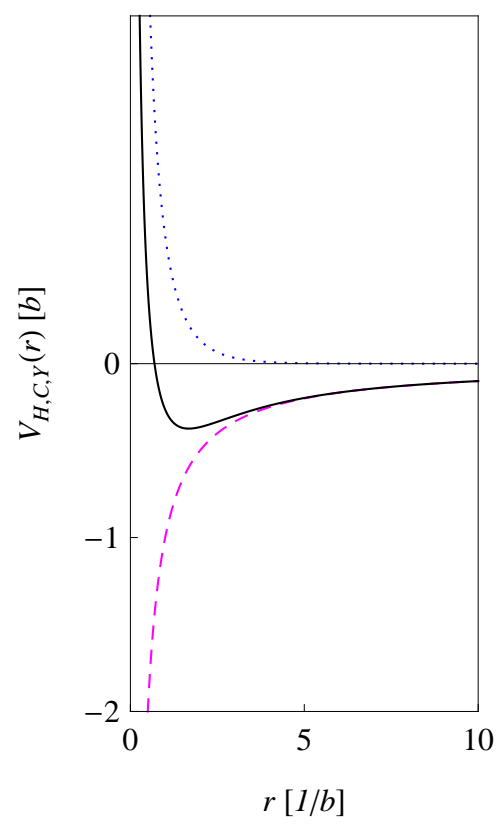

(f)

Figure 1: Hellmann potential $V_{\mathrm{H}}(r)$ (black solid lines), defined by the superposition (2) of a (by assumption attractive) Coulomb potential $V_{\mathrm{C}}(r)$ (dashed magenta lines) and a Yukawa potential $V_{\mathrm{Y}}(r)$ (dotted blue lines), for a fixed value $\kappa=1$ of the Coulomb coupling, and six choices, $v=10$ (a), $v=1$ (b), $v=0.5$ (c), $v=-0.5$ (d), $v=-1$ (e), and $v=-2$ (f), of the Yukawa coupling, in one-to-one correspondence representative of the size relationships (3).

\section{Boundedness from Below: Lower Operator Bounds}

Before being entitled to talk about bound states controlled by a Hamiltonian $H$, one should feel obliged to establish the appropriate semiboundedness of the operator under discussion, defined, for given kinetic term $T(\boldsymbol{p})$, by the behaviour of the involved interaction potential, in our case of the Hellmann potential $V_{\mathrm{H}}(r)$. Unsurprisingly, the latter potential is bounded from below by a Coulomb-type term with suitably chosen coupling strength. Depending on whether the Yukawa contribution to $V_{\mathrm{H}}(r)$ is positive or negative, we distinguish two cases: 


$$
\begin{array}{llll}
V_{\mathrm{H}}(r) \geq-\frac{\kappa+v}{r}, & \text { for } \quad v>0 & \Longleftrightarrow & V_{\mathrm{Y}}(r)<0, \\
V_{\mathrm{H}}(r) \geq-\frac{\kappa}{r}, & \text { for } \quad v \leq 0 & \Longleftrightarrow & V_{\mathrm{Y}}(r) \geq 0 .
\end{array}
$$

For Yukawa couplings in the range $v \leq-\kappa$ entailing the positivity of the Yukawa potential, this Coulomb lower bound is clearly far from optimum and consequently easily improvable:

- For $v<-\kappa$, the Hellmann potential is trivially bounded from below by its minimum,

$$
V_{\mathrm{H}}(r) \geq \min _{0 \leq r<\infty} V_{\mathrm{H}}(r)=V_{\mathrm{H}}(\underline{r})>-\infty .
$$

- For $v=-\kappa$, the Hellmann potential is bounded from below by its value at the origin,

$$
V_{\mathrm{H}}(r) \geq V_{\mathrm{H}}(0)=v b=-\kappa b .
$$

For $v>-\kappa$, we may take advantage of available knowledge about the spectral properties of the semirelativistic Hamiltonian $H$ for Coulomb-type interaction potentials $V_{\mathrm{C}}(r)$ with yet to be constrained strength $\kappa$; the eigenvalue equation of this operator $H$ defines the spinless relativistic Coulomb problem. The relevant quintessence of spectral insights [13,14] is that,

- for $V(\boldsymbol{x})=V_{\mathrm{C}}(r)$, the Hamiltonian $H$ is essentially self-adjoint for Coulomb coupling

$$
\kappa \leq 1
$$

- if $V(\boldsymbol{x})=V_{\mathrm{C}}(r)$, the Hamiltonian $H$ has a Friedrichs extension for Coulomb coupling

$$
\kappa \leq \kappa_{\mathrm{c}}=\frac{4}{\pi}=1.273239 \ldots ;
$$

- for $V(\boldsymbol{x})=V_{\mathrm{C}}(r)$, the spectrum of the Hamiltonian $H, \sigma(H)$, is bounded from below:

$$
\sigma(H) \geq 2 m \sqrt{1-\left(\frac{\kappa}{\kappa_{\mathrm{c}}}\right)^{2}}=2 m \sqrt{1-\left(\frac{\pi \kappa}{4}\right)^{2}} \quad \text { if and only if } \quad \kappa \leq \kappa_{\mathrm{c}} .
$$

Restricting the admissible interval of couplings $\kappa$ yields an improvement of this bound [15]:

$$
\sigma(H) \geq 2 m \sqrt{\frac{1+\sqrt{1-\kappa^{2}}}{2}} \quad \text { for } \quad \kappa \leq 1 .
$$

So, in conclusion, we managed to deduce a first firm intermediate result: By limiting, where necessary, the magnitude of both coupling parameters involved in the interaction potential, the spinless relativistic Hellmann problem under study may be formulated in a well-defined manner. Phrased differently, it can be defined in such a way that its spectrum is guaranteed to be bounded from below, irrespective of the range parameter entering in its Yukawa term.

By evaluating expectation values of the Hamiltonian $H$ over cleverly chosen trial states, it is a rather easy task both to convince oneself of the necessity of constraining those among the involved interaction strengths that - by contributing to a negative singularity of $V_{\mathrm{H}}(r)$ at the origin - otherwise will spoil the desired boundedness from below of the Hamiltonian $H$ and to provide (more or less satisfactory) estimates for upper bounds to those couplings. For all potentially problematic Yukawa coupling strengths $v>-\kappa$, this is demonstrated by adopting a rather simple trial state defined, in either configuration $(r \equiv|\boldsymbol{x}|)$ or momentum $(p \equiv|\boldsymbol{p}|)$ space, by the normalized radial function (enjoying a variational parameter $\mu>0$ ) 


$$
\phi(r)=2 \mu^{3 / 2} \exp (-\mu r) \quad \Longleftrightarrow \quad \widetilde{\phi}(p)=\frac{(2 \mu)^{5 / 2}}{\sqrt{\pi}} \frac{1}{\left(p^{2}+\mu^{2}\right)^{2}} .
$$

The implied expectation value of the kinetic term $T(\boldsymbol{p})$ may be given once and forever $[8,9]$ :

$$
\begin{aligned}
\langle T(\boldsymbol{p})\rangle & =\frac{4}{3 \pi\left(m^{2}-\mu^{2}\right)^{5 / 2}} \\
& \times\left[\mu \sqrt{m^{2}-\mu^{2}}\left(3 m^{4}-4 m^{2} \mu^{2}+4 \mu^{4}\right)+3 m^{4}\left(m^{2}-2 \mu^{2}\right) \sec ^{-1}\left(\frac{m}{\mu}\right)\right] .
\end{aligned}
$$

Likewise, that of any sufficiently simple potential, such as $V_{\mathrm{H}}(r)$, can be found analytically:

$$
\left\langle V_{\mathrm{H}}(r)\right\rangle=-\kappa \mu-\frac{4 v \mu^{3}}{(b+2 \mu)^{2}} .
$$

By Laurent expansion in $1 / \mu$, the emerging expectation value of $H$ behaves, for large $\mu$, like

$$
\begin{aligned}
\langle H\rangle & \equiv\langle T(\boldsymbol{p})\rangle+\left\langle V_{\mathrm{H}}(r)\right\rangle \\
& =\left(\frac{16}{3 \pi}-\kappa-v\right) \mu+v b+O\left(\frac{1}{\mu}\right) \underset{\mu \rightarrow \infty}{\longrightarrow}-\infty \quad \text { for } \quad \kappa+v>\frac{16}{3 \pi} .
\end{aligned}
$$

Mitigating the Coulombic singularity of $V_{\mathrm{H}}(r)$ at the origin needed for a finite ground-state energy thus requires the sum of Coulomb and Yukawa couplings to satisfy an upper bound,

$$
\kappa+v \leq \frac{16}{3 \pi}=1.69765 \ldots
$$

that can be sharpened by discriminating with respect to the sign of our Yukawa coupling $v$ :

$$
\begin{array}{lll}
\kappa+v \leq \frac{16}{3 \pi} & \text { for } & v=|v|>0, \\
\kappa \leq \frac{16}{3 \pi}+|v| & \text { for } & -\kappa<v=-|v|<0 .
\end{array}
$$

\section{Delimiting Spinless-Salpeter Bound-State Counts?}

Another set of questions entering one's mind concerns the actual number of bound states of spinless Salpeter equations. Is this number nonvanishing? Does there exist a bound state at all? Is this number finite or infinite? A couple of considerations may shed light on this issue:

- For the Hamiltonian (1) with relativistic kinetic term $T(\boldsymbol{p})$, an overwhelming amount of knowledge about the number of its bound states does not seem to exist. The bound found in Ref. [18] holds for every nonpositive interaction potential $V(\boldsymbol{x})$ that satisfies

$$
V(\boldsymbol{x}) \in L^{3 / 2}\left(\mathbb{R}^{3}\right) \cap L^{3}\left(\mathbb{R}^{3}\right) .
$$

Consequently, this latter finding is straightforwardly applicable to the Woods-Saxon potential [6] or the kink-like potential [10] but not to the Hellmann potential (2) since

$$
V_{\mathrm{H}}(r) \notin L^{3 / 2}\left(\mathbb{R}^{3}\right) \cap L^{3}\left(\mathbb{R}^{3}\right) .
$$


- For any potential $V(\boldsymbol{x})$, the operator inequality fulfilled by the kinetic term $T(\boldsymbol{p})$ and its nonrelativistic (NR) limit $T_{\mathrm{NR}}(\boldsymbol{p})$ translates, via the arising Hamiltonians, into an inequality for the discrete eigenvalues at the bottom of the associated energy spectra: The relativistic kinetic energy $T(\boldsymbol{p})$ is bounded from above by its nonrelativistic limit,

$$
T(\boldsymbol{p}) \leq T_{\mathrm{NR}}(\boldsymbol{p}) \equiv 2 m+\frac{\boldsymbol{p}^{2}}{m} .
$$

So, the spinless-Salpeter Hamiltonian $H$ and its Schrödinger counterpart $H_{\mathrm{NR}}$ satisfy

$$
H \leq H_{\mathrm{NR}} \equiv T_{\mathrm{NR}}(\boldsymbol{p})+V(\boldsymbol{x}) \text {. }
$$

A spectral comparison theorem, reviewed, e.g., in Refs. [19-23], then allows us to cast this operator inequality into a relation among the corresponding ordered eigenvalues:

$$
E_{k} \leq E_{k, \mathrm{NR}}, \quad k \in \mathbb{N}_{0} .
$$

Hence, to every discrete bound state of $H_{\mathrm{NR}}$ corresponds a discrete bound state of $H$. The number of bound states of $H$ thus cannot be less than that of the respective $H_{\mathrm{NR}}$.

- Every such Schrödinger Hamiltonian with interaction potential (2) of Hellmann type, in turn, upon being confronted with suitable Coulomb problems, proves to support at least one discrete bound state, irrespective of the value of the Yukawa coupling $v$ [24].

- Owing to the comparatively fast decay of the exponential in its Yukawa contribution, the behaviour of any Hellmann potential approaches, in the limit of large distances $r$, the behaviour of its Coulomb contribution. For a nonrelativistic Hamiltonian $H_{\mathrm{NR}}$, in turn, the approach of an attractive Coulomb-like potential to zero in the limit $r \rightarrow \infty$ is definitely not fast enough to constrain the number of bound states to a finite value. This circumstance should be and is reflected by existing upper bounds on the number of nonrelativistic bound states. For instance, the limit of Ref. [25] diverges since, for a Hellmann potential, the integral entering this finding as the key quantity behaves like

$$
\int_{0}^{R} \mathrm{~d} r r\left|\widetilde{V}_{\mathrm{H}}(r)\right| \underset{R \rightarrow \infty}{\longrightarrow} \infty, \quad \widetilde{V}_{\mathrm{H}}(r) \equiv-\max \left[0,-V_{\mathrm{H}}(r)\right] .
$$

In summary, our tentative conclusion must be that (as consequence of the predominance of the Coulomb potential at sufficiently large distances) the comparatively slow Coulomb-like approach to zero of any Hellmann potential causes - in contrast to the Yukawa case - the number of bound states of a spinless relativistic Hellmann problem to grow beyond bounds.

\section{Variational Upper Limits on Bound-State Energies}

Given the lower bounds of Sec. 3, we next aim at constricting, as tight as possible, the exact eigenvalues of the semirelativistic Hamiltonian $H$ with generalized Hellmann potential (2).

A main tool for a rough localization of the discrete spectrum of a Hilbert-space operator $H$, consisting of all isolated eigenvalues of finite multiplicity below the onset of the essential spectrum, is the Rayleigh-Ritz variational technique provided by the minimum-maximum theorem [26]: The lowest-lying $d$ among the eigenvalues, $E_{k}, k \in \mathbb{N}_{0}$, assumed to be ordered according to $E_{0} \leq E_{1} \leq \cdots$, of a self-adjoint operator $H$ bounded from below are bounded from above by the $d$ eigenvalues, $\widehat{E}_{k}, k=0,1, \ldots, d-1$, assumed to be ordered according to $\widehat{E}_{0} \leq \widehat{E}_{1} \leq \cdots \leq \widehat{E}_{d-1}$, of the restriction of $H$ to any $d$-dimensional subspace of its domain, 


$$
E_{k} \leq \widehat{E}_{k} \quad \forall \quad k=0,1, \ldots, d-1
$$

For a spherically symmetric interaction potential $V(\boldsymbol{x})=V(r)$, each basis vector of the finite-dimensional trial space setting the stage for application of this variational framework may be conveniently represented by a product of a radial function and a spherical harmonic $\mathcal{Y}_{\ell m}(\Omega)$ for orbital angular momentum and associated projection quantum numbers $\ell \in \mathbb{N}_{0}$ and $m=\ell, \ell-1, \ldots,-\ell$, depending on the solid angle $\Omega$. An orthonormal basis of this kind, rather well suited for tackling on analytical grounds bound-state problems whose definition comprises the square-root operator of the relativistic kinetic energy, utilizes two variational parameters: $\mu$, with dimension of mass and satisfying $\mu \in(0, \infty)$, and $\beta$, dimensionless and satisfying $\beta \in\left(-\frac{1}{2}, \infty\right)$. It can be given analytically in configuration and momentum space, in the former by generalized-Laguerre orthogonal polynomials $L_{k}^{(\gamma)}(x)$ [27] for parameter $\gamma$ :

$$
\begin{aligned}
& \psi_{k, \ell m}(\boldsymbol{x})=\sqrt{\frac{(2 \mu)^{2 \ell+2 \beta+1} k !}{\Gamma(2 \ell+2 \beta+k+1)}} r^{\ell+\beta-1} \exp (-\mu r) L_{k}^{(2 \ell+2 \beta)}(2 \mu r) \mathcal{Y}_{\ell m}\left(\Omega_{\boldsymbol{x}}\right), \\
& L_{k}^{(\gamma)}(x) \equiv \sum_{t=0}^{k}\left(\begin{array}{c}
k+\gamma \\
k-t
\end{array}\right) \frac{(-x)^{t}}{t !}, \quad k=0,1,2, \ldots, \\
& \int \mathrm{d}^{3} x \psi_{k, \ell m}^{*}(\boldsymbol{x}) \psi_{k^{\prime}, \ell^{\prime} m^{\prime}}(\boldsymbol{x})=\delta_{k k^{\prime}} \delta_{\ell \ell^{\prime}} \delta_{m m^{\prime}} .
\end{aligned}
$$

Fourier transformation immediately provides the momentum-space representation of these basis states; explicit expressions of these functions can be found in, e.g., Refs. [6,8,9,20,23].

Table 1 illustrates the application of the variational technique recalled above to spinless relativistic Hellmann problems by presenting the set of upper limits on the binding energies

$$
B_{k} \equiv E_{k}-2 m, \quad k=0,1,2, \ldots, d-1,
$$

derived, for representatives of three of our six categories of generalized Hellmann potentials identified by Eqs. (3), for a kind of self-suggesting choice of parameter values and optimized (i.e., reasonably chosen) trial-space dimension $d \in\{29,30,31\}$. Enlarging the trial space as well as exploiting one's freedom to vary the two parameters $\mu$ and $\beta$ clearly should allow for an improvement of any such upper limits. A dash in Table 1 does not necessarily imply that the associated bound state does not exist. Rather, it should be understood as an indication that, within the adopted setting, no trustable upper limit (apart from zero) could be found.

\section{Exploiting Hellmann Potentials: Rules of the Game}

For the notationally simpler case of equal-mass constituents, we sketched the boundaries to be expected for bound-state spectra of spinless relativistic Hellmann problems; with a little more effort, the generalization to unequal masses is straightforward. These findings should, for future Hellmann studies, reduce the risk to get lost in the vast jungle of approximations.

Since recently, interest in the solutions of the spinless relativistic Hellmann problem can be spotted in the literature. At present, we are aware of just two attempts [28,29] to exploit for that purpose a longstanding but very approximate way of dealing with spinless Salpeter equations. Unfortunately, from our point of view this latter approach is very problematic in (at least) two well-known respects: On the one hand, as a first step towards treatability, the 
Table 1: Variational upper bounds, in units of the identical mass, $m$, of the two bound-state constituents, on the binding energies (8) of the low-lying bound states of generic relativistic Hellmann problems (identifying any bound state by radial quantum number $n_{r}$ and orbital angular momentum quantum number $\ell$ ), derived by relying on the trial-space basis $(7)$ and employing, as numerical input values, for the two variational parameters $\beta=1$ and $\mu=m$, for the Yukawa range parameter $b=m$, and three illustrative combinations of the coupling strengths: $\kappa=v=\frac{1}{2}$, exemplifying all set-(3b) potentials; $\kappa=1$ and $v=-1$, exemplifying all set-(3e) potentials; $\kappa=1$ and $v=-2$, exemplifying all set-(3f) potentials. (To facilitate appreciation, the spectral lower bounds arising from Eqs. (6), (5), or (4) are also provided.)

\begin{tabular}{|c|c|c|c|c|}
\hline \multicolumn{2}{|c|}{ Bound state } & \multirow{2}{*}{$\begin{array}{l}\kappa=v=\frac{1}{2} \\
{[\operatorname{case}(3 \mathrm{~b})]}\end{array}$} & \multirow{2}{*}{$\begin{array}{c}\kappa=1, v=-1 \\
{[\text { case }(3 \mathrm{e})]}\end{array}$} & \multirow{2}{*}{$\begin{array}{c}\kappa=1, v=-2 \\
{[\text { case }(3 \mathrm{f})]}\end{array}$} \\
\hline$n_{r}$ & $\ell$ & & & \\
\hline \multirow{2}{*}{\multicolumn{2}{|c|}{$\begin{array}{r}\text { Spectral lower bound } \\
\sigma(H)[m]\end{array}$}} & [Eq. (6)] & {$[\mathrm{Eq} .(5)]$} & {$[\mathrm{Eq} .(4)]$} \\
\hline & & -0.58578 & -1 & -0.37336 \\
\hline 0 & 0 & -0.11673 & -0.17951 & -0.14410 \\
\hline 0 & 1 & -0.01579 & -0.06294 & -0.06157 \\
\hline 0 & 2 & -0.00616 & -0.02813 & -0.02812 \\
\hline 0 & 3 & - & -0.01553 & -0.01553 \\
\hline 1 & 0 & -0.02107 & -0.05464 & -0.04786 \\
\hline 1 & 1 & -0.00509 & -0.02810 & -0.02762 \\
\hline 1 & 2 & - & -0.01482 & -0.01481 \\
\hline 1 & 3 & - & -0.00624 & -0.00624 \\
\hline 2 & 0 & -0.00688 & -0.02566 & -0.02338 \\
\hline 2 & 1 & - & -0.01391 & -0.01356 \\
\hline 2 & 2 & - & -0.00122 & -0.00120 \\
\hline 3 & 0 & - & -0.01104 & -0.00840 \\
\hline
\end{tabular}

procedure relies on an expansion of the square-root operator of any relativistic kinetic term $T(\boldsymbol{p})$ in powers of $\boldsymbol{p}^{2} / \mathrm{m}^{2}$ and subsequent truncation of the expansion at a convenient order. The pseudo-spinless-Salpeter Hamiltonian emerging from such mistreatment, however, can be shown [6] to be an operator unbounded from below for all interaction potentials that are not too singular at the spatial origin, including all generalized Hellmann potentials. On the other hand, as a second step, the formulation of an equation of Schrödinger type is enforced by reinsertion of that eigenvalue equation of the pseudo-spinless-Salpeter Hamiltonian that holds for its lower-order truncation. The latter manipulation too is known to be flawed, due to an evidently inappropriate neglect of a commutator term [30] in its derivation. In view of these deficiencies, it does not make sense to discuss the results of Refs. [28,29] in any detail.

\section{References}

[1] H. A. Bethe and E. E. Salpeter, Phys. Rev. 82 (1951) 309.

[2] M. Gell-Mann and F. Low, Phys. Rev. 84 (1951) 350. 
[3] E. E. Salpeter and H. A. Bethe, Phys. Rev. 84 (1951) 1232.

[4] W. Lucha and F. F. Schöberl, Phys. Rev. Lett. 64 (1990) 2733.

[5] W. Lucha, Mod. Phys. Lett. A 5 (1990) 2473.

[6] W. Lucha and F. F. Schöberl, Int. J. Mod. Phys. A 29 (2014) 1450057, arXiv:1401.5970 [hep$\mathrm{ph}]$.

[7] W. Lucha and F. F. Schöberl, EPJ Web Conf. 80 (2014) 00049, arXiv:1407.4624 [hep-ph].

[8] W. Lucha and F. F. Schöberl, Int. J. Mod. Phys. A 29 (2014) 1450181, arXiv:1408.4957 [hep$\mathrm{ph}]$.

[9] W. Lucha and F. F. Schöberl, Int. J. Mod. Phys. A 29 (2014) 1450195, arXiv:1410.5241 [hep$\mathrm{ph}]$.

[10] W. Lucha and F. F. Schöberl, Int. J. Mod. Phys. A 30 (2015) 1550062, arXiv:1412.4950 [hep$\mathrm{ph}]$.

[11] H. Hellmann, J. Chem. Phys. 3 (1935) 61.

[12] H. Hellmann and W. Kassatotschkin, J. Chem. Phys. 4 (1936) 324.

[13] I. W. Herbst, Commun. Math. Phys. 53 (1977) 285.

[14] I. W. Herbst, Commun. Math. Phys. 55 (1977) 316.

[15] A. Martin and S. M. Roy, Phys. Lett. B 233 (1989) 407.

[16] J. C. Raynal, S. M. Roy, V. Singh, A. Martin, and J. Stubbe, Phys. Lett. B 320 (1994) 105.

[17] W. Lucha and F. F. Schöberl, J. Math. Phys. 41 (2000) 1778, arXiv:hep-ph/9905556.

[18] I. Daubechies, Commun. Math. Phys. 90 (1983) 511.

[19] W. Lucha and F. F. Schöberl, Phys. Rev. A 54 (1996) 3790, arXiv:hep-ph/9603429.

[20] W. Lucha and F. F. Schöberl, Int. J. Mod. Phys. A 14 (1999) 2309, arXiv:hep-ph/9812368.

[21] W. Lucha and F. F. Schöberl, Fizika B 8 (1999) 193, arXiv:hep-ph/9812526.

[22] W. Lucha and F. F. Schöberl, J. Math. Phys. 41 (2000) 1778, arXiv:hep-ph/9905556.

[23] W. Lucha and F. F. Schöberl, Recent Res. Dev. Phys. 5 (2004) 1423, arXiv:hep-ph/0408184.

[24] R. L. Hall and Q. D. Katatbeh, Phys. Lett. A 287 (2001) 183, arXiv:math-ph/0107015.

[25] V. Bargmann, Proc. Natl. Acad. Sci. USA 38 (1952) 961.

[26] M. Reed and B. Simon, Methods of Modern Mathematical Physics IV: Analysis of Operators (Academic Press, New York, 1978).

[27] M. Abramowitz and I. A. Stegun (eds.), Handbook of Mathematical Functions (Dover, New York, 1964).

[28] A. D. Antia and E. E. Ituen, Ann. Univ. Mar. Curie-Skłodowska (Lublin) 71(AAA) (2016) 53.

[29] A. Arda, Indian J. Phys. 91 (2017) 903; arXiv:1701.01336 [physics.gen-ph].

[30] Y. Chargui, Eur. Phys. J. Plus 133 (2018) 543. 